\title{
Sensor-Assisted Global Motion Estimation for Efficient UAV Video Coding
}

\author{
Yang $\mathrm{Mi}^{\dagger}$, Chunbo Luo ${ }^{\dagger}$, Geyong $\mathrm{Min}^{\dagger}$, Wang $\mathrm{Miao}^{\dagger}$, Liang $\mathrm{Wu}^{\ddagger}$, and Tianxiao Zhao ${ }^{+}$ \\ $\dagger$ College of Engineering, Mathematics and Physical Sciences, University of Exeter, UK \\ $\ddagger$ Research Department of Multimedia, Huawei, China \\ + School of Information and Electronics, Beijing Institute of Technology, China \\ E-mail: \{ym310, c.luo, g.min, wang.miao\}@exeter.ac.uk, liang.wu@huawei.com, \\ chirs90@126.com
}

\begin{abstract}
In this paper, we propose a novel video coding scheme to significantly reduce the coding complexity and enhance overall coding efficiency in videos acquired by high mobility devices such as unmanned aerial vehicles (UAVs). In order to reduce the encoded data bits and encoding time to facilitate real-time data transmission, as well as minimize the image distortion caused by the jitter of onboard camera, a sensor-assisted global motion estimation (GMV) algorithm is designed to calculate perspective transformation model and global motion vectors, which are used in both the interframe coding to improve the coding efficiency and intraframe coding to reduce block search complexity. We conducted comprehensive simulation experiments on official HM-16.10 codec and the performance results show the proposed method can achieve faster block search by $50 \%$ to $60 \%$ speedup and lower bitrate by $15 \%$ to $30 \%$ compared with standard HEVC coding software.
\end{abstract}

Index Terms-HEVC, sensor-assisted global motion estimation, low delay

\section{INTRODUCTION}

In recent years, one of the predominate focuses of wireless communication systems is the support of multimedia services. The use of UAVs equipped with high definition cameras is rapidly growing in the tasks such as video reconnaissance, exploitation, and surveillance. In the near future, video stream will account for over $80 \%$ of consumer Internet traffic according to [1]. The using of ultra high definition videos with high framerate and multi view will raise new challenges to video transmission tasks. Therefore, it is vital to investigate efficient video compression algorithms for wireless multimedia services.

H.265/HEVC is the latest video coding technology whose predecessor is H.264/AVC. In benchmark video compression standards, such as H.265/HEVC, global motion estimation (GME) is not adopted due to its suboptimal rate-distortion performance and complexity. The motion of each coding unit in UAV video stream is composed of camera and foreground object motion. GME is a technique that attempts to find the perceptive projection matrix between two images for video processing of high-mobility systems [2]. Differing from region ME which attempts to find the corresponding position of each individual pixel in its reference pictures, GME identifies the background motion introduced by the camera to obtain a stable and smooth video. GME requires much cheaper computation compared with local ME (LME), but can achieve high resolution image compression and transmission [3].

Image-based GME has already been integrated in MPEG4 verification model [4] which supports translation motion. The authors of [5] suggested that the motion information can be used to merge blocks. A sensors-aided video encoding method $(\mathrm{SaVE})$ that calculates the rotation movement of the camera for H.264 is proposed [6], and this method outperforms standard H.264 by $27 \%$ speedup. An approach called Sensor-assisted Motion Estimation (SaME) was proposed to estimate the global linear displacement [7], SaME focus on linear displacement estimation. A low-complexity video encoder using affine model and a matched decoder is proposed to get rid of block-level motion estimation within group of pictures (GOP) [3]. This method has proved to be outstanding in reducing bitrates but sacrifices video quality.

For many UAV videos, large translation and rotation and scaling exist between adjacent frames. The image frames might move out of the search window or image distortion caused by rotation/zoom might result in prediction unit block matching algorithms failure. The compression ratio deteriorates significantly if the block matching algorithm fails. To provide a global motion model that fits a wide range of mid-altitude UAVs, the authors of [2] propose to derived image coordinate system transform model from metadata. A low delay and low complexity video encoding for UAV inspection application is presented in [8] which replace inter-frames using homography matrix. Authors of [9], [10] also use metadata to build georeferencing model for midaltitude fixed-wing UAVs image geometric correlation.

The major contribution of this paper includes: First, a novel method for fast motion vector prediction is proposed, and the sensor-assisted GME method is implemented based on HM-16.10 software. Second, we confirm that by performing perspective transformation, the multiscale structural similarity (MS-SSIM) between adjacent frames is increased. And last, we built our UAV video dataset with corresponding sensor log information which dataset provides an important source for future sensor assisted video codec research.

The remaining of the paper is as follows: Section II introduces the sensor-assisted video coding software framework and our method, the using of perspective transformation model and global motion vectors. Section III provides experiment results to study the performance of our framework 


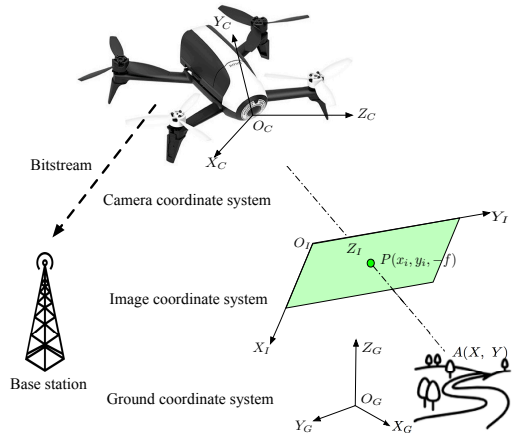

Fig. 1: The wireless communication scenario of our sensorassisted video coding method and pinhole model.

\begin{tabular}{l|l} 
Name & Description \\
\hline $\mathrm{X}$ speed $v_{x}$ & Horizontal speed (X-axis), in yard \\
Y speed $v_{y}$ & Horizontal speed (Y-axis), in yard \\
$\mathrm{Z}$ speed $v_{z}$ & Vertical speed, in yard \\
Vehicle roll $\theta$ & Euler angle (X-axis), in radians \\
Vehicle pitch $\phi$ & Euler angle (Y-axis), in radians \\
Vehicle heading $\psi$ & Euler angle (Z-axis)n, in radians \\
Altitude $d$ & Terrain height, in centimeters \\
\hline
\end{tabular}

TABLE I: Sensor parameters used in the algorithm.

under different QPs and compares the overall performance with standard HM-16.10. Finally, Section IV concludes this paper.

\section{Methodology}

\section{A. System model}

The deployment scenario is illustrated in Fig. 1 which shows a small UAV with onboard camera and sensors flying around a base station within transmission range. Video streams are captured by the onboard camera, while UAV and camera motions are recorded by its sensors. The raw video stream is encoded before being transmitted to the receiver via wireless channel.

Fig. 2 describes the flow diagram of the proposed methodology. The encoder takes video stream and corresponding sensor log as inputs. The perspective transformation model (homography matrix) between image coordinate system and ground coordinate system is computed and updated once new frame and sensor data arrive. Raw image frames firstly undergo perspective transformation to remove undesired distortions caused by external motion. The GME method completely relies on sensor information provided by UAV system. An frame motion monitor is used in order to determine whether large-scale motion exists between two adjacent frames, if not, the encoding process could be skipped by transmitting a 9-element homography matrix (HM) instead. Otherwise, block matching process with fast motion vector predictor (MVP) candidate list is executed to locate the best local MV. Table. I shows the sensor data used in our method.

\section{B. Perspective transformation for UAV videos}

The global motion of UAV video can be modelled by a combination of motion of the UAV and camera. To eliminate the distortion caused by those movements, a projection model is necessary for representing the relationship of image coordinate system and ground coordinate system.

The HM is modelled with intrinsic and extrinsic parameters. Intrinsic parameters describe the mapping between camera coordinates and image coordinates in the image frame, while extrinsic parameters define the location and orientation of the camera coordinate with respect to the ground coordinate (See Fig. 1).

$$
K=\left[\begin{array}{ccc}
\frac{1}{s_{x}} & 0 & c_{x} \\
0 & \frac{1}{s_{y}} & c_{y} \\
0 & 0 & 1
\end{array}\right]
$$

where $\left(s_{x}, s_{y}\right)$ is the width and height of a single pixel, $\left(c_{x}, c_{y}\right)$ is the horizontal and vertical offsets of camera.

Translation of the UAV between two time instances can be obtained by constructing an extrinsic matrix. We assume that the camera rotation and translation of $F(t+1)$, with respect to $F(t)$, is denotes as $R$ and $T$, respectively. The rotation matrix between image coordinate system and ground coordinate system can thus be obtained as

$$
R=R_{\psi} R_{\phi} R_{\theta}
$$

where $R_{\psi}, R_{\phi}$, and $R_{\theta}$ denote heading rotation, pitch rotation and roll rotation, respectively.

The displacement between image coordinate system and ground coordinate system is represented as

$$
T=\left[\begin{array}{lll}
t_{x} & t_{y} & t_{z}
\end{array}\right]^{\prime}
$$

Hence, the perspective transformation at time $t$ is defined as follow:

$$
H_{t}=K^{-1}\left(R+\frac{1}{d} T\right) K
$$

where $d$ denotes the altitude.

Hence, the calibrated image pair is expressed as follows

$$
\begin{gathered}
F_{t}^{*}=H_{t} F_{t} \\
F_{t+1}^{*}=H_{t+1} F_{t+1}
\end{gathered}
$$

\section{Fast Motion Vector Predictor Estimation}

After coordination transformation, the global displacement of the UAV simply obeys a linear equation $D=$ [dx $d y]^{\prime}$, where $d_{x}$ and $d_{y}$ denote the GMV at time $t$.

$$
D^{*}=\left[\begin{array}{ll}
d x^{*} & d y^{*}
\end{array}\right]^{\prime}=\left[\frac{d x}{s_{x}} \frac{d y}{s_{y}}\right]^{\prime}
$$

The translation between frame $F(t)$ and frame $F(t+1)$ can be expressed as follows

$$
F_{t+1}=H_{t+1}^{-1} D H_{t} F_{t}=M F_{t}
$$

In our software diagram, an image monitor based on the idea of SSIM is placed before the prediction module as shown in Fig. 3. Assuming Ftmp $=M F_{t}$, the SSIM between Ftmp and $F_{t+1}$ is calculated. If SSIM satisfies a given threshold, then $F_{t+1}$ will not be processed by the encoder, but 9-element $H_{t+1}$ will be transmitted instead. 


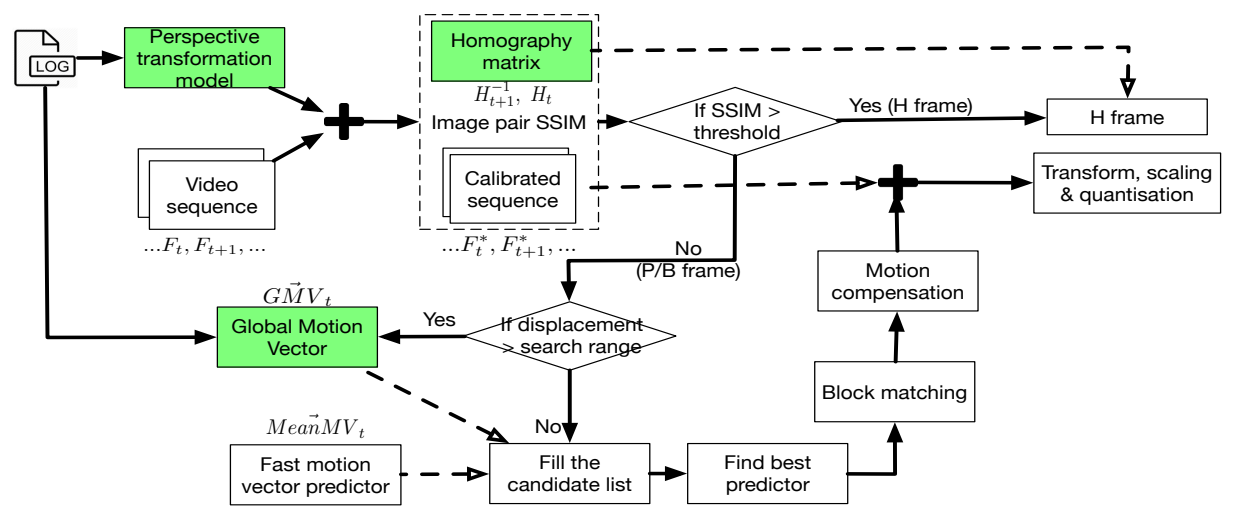

Fig. 2: The flow diagram of the proposed sensor-assisted video coding approach.

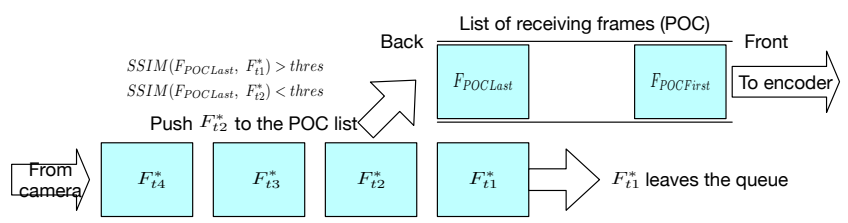

Fig. 3: The dropping out process. If two adjacent frames are considered to be similar enough, the new frame will not be processed by the encoder.

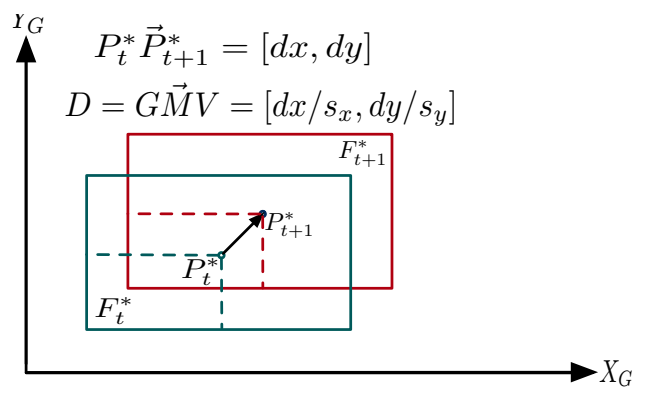

Fig. 4: The method of GMV calculation for the current block, where $P^{*}$ denotes the central point of a frame.

The frame $F_{t+1}$ can be reconstructed from $H_{t}, F_{t}$ and $H_{t+1}$ at the decoder end.

If the sensor information as well as the multiplication are absolutely accurate, $M$ can represent the global motion from $F_{t}$ to $F_{t+1}$, and thus $F_{t}^{*}$ is similar to $F_{t+1}$. However, due to the UAV sensor measurement error and local motion existing between block range, in fact in most of the cases $M$ cannot represent the best motion for each block. Therefore, the encoder has to execute block matching algorithms to find the best candidate MVs for those blocks.

We notice that in our aerial video coding process, coding blocks belonging to the background area tend to share MVs pointing to a same directory. This means that those blocks follows the global motion and the best MV of a current block has strong correlation to its spatial neighbours. Also, the blocks belonging to the foreground objects are likely to have similar LMVs of their decoded neighbours. Inspired by the work [11], we implement our fast ME process within two steps, initial candidate list and block matching search. Similar to the candidate list used in standard HEVC, our

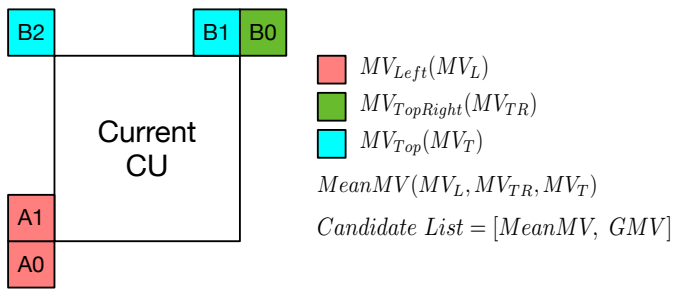

Fig. 5: The example of choosing spatial MVs from neighbouring blocks and filling the candidate list for the current block.

candidate list is filled up by two MVPs, one is the GMV from sensor information which represents the temporal correlation between reference frame and current frame (See Fig. 4), the other is a mean of neighbouring MVs (as shown in Fig. 5).

$$
\text { MeanMV }=\operatorname{Mean}\left(\overrightarrow{M V}{ }_{L}, \overrightarrow{M V} V_{T R}, \overrightarrow{M V} V_{T}\right)
$$

If the total elements in candidate list is less than two (for instance, spatial MVs are not available), a zero $\operatorname{MV}(0,0)$ will be added to fill the candidate list. Thus, the candidate MV list (if both temporal MV and spatial MV are available) is shown as

Candidate_List $= \begin{cases}\overrightarrow{M V}=D, & \text { GMV } \\ \overrightarrow{M V} V_{1}=M e a n M V, & \text { LMV }\end{cases}$

Fig. 5 shows the neighbouring blocks (on the left, up, and up-right) of the current block and the MVs are denotes as $\overrightarrow{M V} V_{L}, \overrightarrow{M V} V_{T R}$, and $\overrightarrow{M V} V_{T}$, respectively. The best predictor is determined by Lagrangian RD cost function, block matching algorithm is performed on the TZSearch window with search range $b w$ after the best MVP is known.

\section{EXPERIMENTS}

The experiments were conducted on a Linux server based on the standard HEVC codec software HM-16.10. Our dataset was divided into four groups, which are $(0,3 \mathrm{~m})$, $(3 m, 6 m),(6 m, 15 m)$, and $(15 m, 30 m)$. The experimental results in low-altitude UAV video indicate that our method are still effective for processing videos collected at higher altitudes. 


\begin{tabular}{|c|c|c|c|c|c|}
\hline & fram & size $(960$ & 576) & & \\
\hline Altitude & QP & YPSNR & YSSIM & Bytes & Time(sec) \\
\hline \multirow{4}{*}{$(0,3 \mathrm{~m})$} & \multirow[b]{2}{*}{22} & 38.5230 & 0.9965 & 30679 & 21.5029 \\
\hline & & 37.8756 & 0.9963 & 29225 & 10.7863 \\
\hline & \multirow[b]{2}{*}{37} & 28.5315 & 0.9495 & 1927 & 9.2817 \\
\hline & & 28.0909 & 0.9428 & 1910 & 4.6961 \\
\hline \multirow{4}{*}{$(3 \mathrm{~m}, 6 \mathrm{~m})$} & \multirow{2}{*}{22} & 37.6262 & 0.9971 & 47709 & 19.7987 \\
\hline & & 37.4478 & 0.9951 & 26344 & 8.2494 \\
\hline & \multirow{2}{*}{37} & 26.5360 & 0.9488 & 3473 & 7.2409 \\
\hline & & 26.9727 & 0.9315 & 2057 & 3.6333 \\
\hline \multirow{4}{*}{$(6 \mathrm{~m}, 15 \mathrm{~m})$} & \multirow{2}{*}{22} & 38.0860 & 0.9954 & 27942 & 19.6222 \\
\hline & & 37.7808 & 0.9939 & 18429 & 6.164 \\
\hline & \multirow{2}{*}{37} & 27.9549 & 0.9339 & 1041 & 8.1853 \\
\hline & & 28.2629 & 0.9357 & 928 & 2.9248 \\
\hline \multirow{4}{*}{$(15 \mathrm{~m}, 30 \mathrm{~m})$} & \multirow{2}{*}{22} & 38.9303 & 0.9952 & 24054 & 20.6611 \\
\hline & & 39.4416 & 0.9956 & 16432 & 6.9023 \\
\hline & \multirow[b]{2}{*}{37} & 28.3769 & 0.9313 & 1554 & 8.4918 \\
\hline & & 30.1734 & 0.9440 & 961 & 2.5265 \\
\hline \multirow{4}{*}{ overall } & \multirow{2}{*}{22} & 38.7784 & 0.9954 & 25288 & 20.6549 \\
\hline & & 38.3579 & 0.9951 & 19790 & 7.2743 \\
\hline & \multirow{2}{*}{37} & 28.8427 & 0.9345 & 1325 & 8.5330 \\
\hline & & 28.7104 & 0.9326 & 1226 & 2.7378 \\
\hline
\end{tabular}

\begin{tabular}{|c|c|c|c|c|c|}
\hline & fram & size $(192$ & × 1080) & & \\
\hline Altitude & QP & YPSNR & YSSIM & Bytes & Time(sec) \\
\hline \multirow{4}{*}{$(0,3 \mathrm{~m})$} & \multirow{2}{*}{22} & 40.4066 & 0.9964 & 121311 & 107.1858 \\
\hline & & 39.2895 & 0.9959 & 100026 & 88.1690 \\
\hline & \multirow{2}{*}{27} & 30.1515 & 0.9535 & 5556 & 102.9819 \\
\hline & & 29.6826 & 0.9355 & 5135 & 37.3786 \\
\hline \multirow{4}{*}{$(3 \mathrm{~m}, 6 \mathrm{~m})$} & \multirow{2}{*}{22} & 39.5452 & 0.9972 & 150474 & 92.4641 \\
\hline & & 38.8273 & 0.9955 & 146783 & 69.3481 \\
\hline & \multirow{2}{*}{37} & 28.2041 & 0.9512 & 10445 & 34.4790 \\
\hline & & 28.7090 & 0.9413 & 6130 & 25.8592 \\
\hline \multirow{4}{*}{$(6 \mathrm{~m}, 15 \mathrm{~m})$} & \multirow{2}{*}{22} & 39.9973 & 0.9957 & 126991 & 87.8458 \\
\hline & & 39.1881 & 0.9945 & 92668 & 54.8097 \\
\hline & \multirow{2}{*}{37} & 29.5621 & 0.9420 & 4066 & 34.3467 \\
\hline & & 29.3384 & 0.9250 & 4479 & 21.4300 \\
\hline \multirow{4}{*}{$(15 \mathrm{~m}, 30 \mathrm{~m})$} & \multirow{2}{*}{22} & 38.2535 & 0.9955 & 115047 & 87.5826 \\
\hline & & 39.3062 & 0.9957 & 98076 & 46.0662 \\
\hline & \multirow{2}{*}{37} & 30.0811 & 0.9409 & 4373 & 43.1807 \\
\hline & & 29.6293 & 0.93142 & 4905 & 19.6568 \\
\hline \multirow{4}{*}{ overall } & \multirow{2}{*}{22} & 39.0130 & 0.9957 & 120602 & 91.6711 \\
\hline & & 39.1977 & 0.9953 & 110838 & 58.0617 \\
\hline & \multirow{2}{*}{37} & 29.8948 & 0.9429 & 4594 & 33.9552 \\
\hline & & 29.3981 & 0.9310 & 5571 & 20.4627 \\
\hline
\end{tabular}

TABLE II: The Video coding results. Results of HM-16.10 are given in the first row (in black) of each group; our results are given in the second row (in blue). YPSNR and YSSIM are used to measure the reconstructed video quality.

\begin{tabular}{|r|l|}
\hline MaxCUHeight & 64 \\
MaxCUWidth & 64 \\
MaxCUDepth & 4 \\
Quantization Parameter (QP) & {$[22,27,32,37]$} \\
Motion Estimation Method & TZSearch \\
Search Range & {$[-16,16]$} \\
Coding Profile & low_delay_main_P \\
\hline
\end{tabular}

TABLE III: Experiment configuration conditions.

The configuration setting can be found in Table. III. To evaluate the performance of our method, we compare the quality achieved by our proposed method (in blue) and HEVC in terms of PSNR, SSIM, average bytes, and average encoding time across all $\mathrm{P}$ frames.

If one or more video frames is ignored by the encoder, the difference between reference frame and current frame accumulates as it can be expected. Table.II demonstrates the summary of encoding results with $\mathrm{H}$ frames. It can be seen that the proposed method efficiently improve the compression ratio while the image quality degradation is small. For instance, with $Q P=22$ and altitude $(6 \mathrm{~m}, 15 \mathrm{~m})$, the average bytes of our sensor-assisted method is $73 \%$ of HM-16.10's, while the PSNR of Y-channel is $0.5 d B$ less than HM-16.10's. The motion of camera contributes to the coding efficiency, with altitude $(3 m, 6 m)$ where the UAV is climbing up swiftly, the coding blocks are divided into more quad-tree blocks in HM-16.10, while the sensor-assisted HM takes advantage of the GMVs in finding matching blocks. With regard to time consumption, our algorithm can save up to $60 \%$ of the inter-frame encoding time of HM16.10's. The second experimental result in Table.II shows that our proposed method is able to cover variety UAV movements at different definitions.

The second experiment compares the encoding performance of HM-16.10 and our method using videos of different framerate and definition (with $Q P=22$ ). The results shown in Fig. 6 suggest that our method supports videos under difference resolution and framerate. In the case of $1920 \times 1080$ videos at $30 \mathrm{fps}$. great benefit of

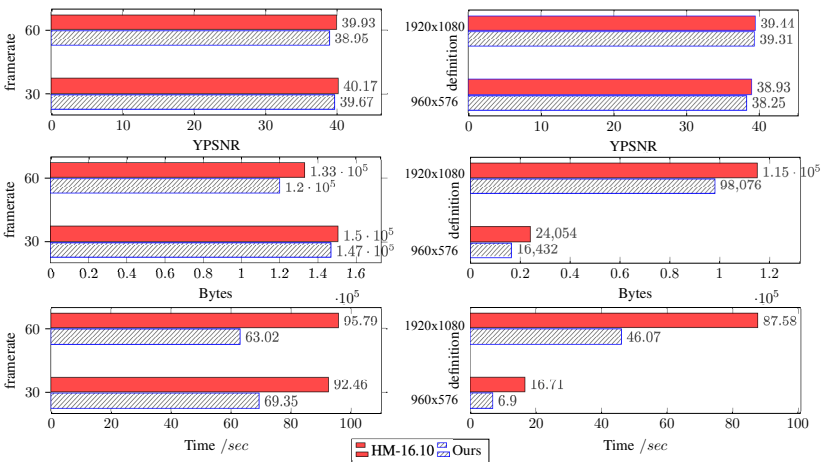

Fig. 6: Left: $1920 \times 1080$ video at $30 \mathrm{fps}$ and $60 \mathrm{fps}$, where the red bar denotes HM-16.10 and the blue pattern bar denotes ours. Right: $960 \times 576$ and $1920 \times 1080$ videos at $30 \mathrm{fps}$, where the red bar denotes HM-16.10 and the blue pattern bar denotes ours.

our method can be observed from the plot, showing $50 \%$ time saving and $15 \%$ bytes saving, at the cost of $-0.5 d B$ YPSNR gain, while compared with HM-16.10's.

\section{Conclusion}

In this paper, we proposed a sensor-assisted global motion estimation method aiming to trade-off reconstructed image quality and encoding time and complexity. Our experiment results show that the proposed method is able to reduce the average encoding time by $50 \%$ to $60 \%$ and the average number of bytes by $15 \%$ to $30 \%$, while the average YPSNR and average YSSIM remain the same level, comparing with the HM-16.10. The proposed method is especially useful for low-delay or nearly real-time video transmission tasks, as well as low power platforms such as small UAVs and wireless sensor nodes in which light computation is essential.

\section{REFERENCES}

[1] A. Asensio, M. Ruiz, and L. Velasco, "Requirements to support cloud, video and $5 \mathrm{~g}$ services on the telecom cloud," in Networks and 
Optical Communications (NOC), 2016 21st European Conference on. IEEE, 2016, pp. 64-69.

[2] H. Li, X. Li, W. Ding, and Y. Huang, "Metadata-assisted global motion estimation for medium-altitude unmanned aerial vehicle video applications," Remote Sensing, vol. 7, no. 10, pp. $12606-$ $12634,2015$.

[3] M. Bhaskaranand and J. D. Gibson, "Low-complexity video encoding for uav reconnaissance and surveillance," in Military Communications Conference, 2011-MILCOM 2011. IEEE, 2011, pp. 16331638.

[4] S. Fukunaga, Y. Nakaya, S. H. Son, and T. Nagumo, "Mpeg-4 video verification model version 16.0," International Organization for Standardization: Coding of Moving Pictures and Audio, vol. 3312, pp. 1-380, 2000.

[5] H. Li, K. Fan, R. Wang, G. Li, and W. Wang, "A motion aided merge mode for hevc," in 2018 IEEE International Conference on Acoustics, Speech and Signal Processing (ICASSP). IEEE, 2018, pp. 1773-1776.

[6] X. Chen, Z. Zhao, A. Rahmati, Y. Wang, and L. Zhong, "Sensorassisted video encoding for mobile devices in real-world environments," IEEE Transactions on Circuits and Systems for Video Technology, vol. 21, no. 3, pp. 335-349, 2011.

[7] L. Areekath and K. K. Palavalasa, "Sensor assisted motion estimation," in Engineering and Systems (SCES), 2013 Students Conference on. IEEE, 2013, pp. 1-6.

[8] R. Zhang, K. Hossain et al., "Low complexity video encoding for uav inspection," in Picture Coding Symposium (PCS), 2016. IEEE, 2016, pp. 1-5.

[9] H. Li, X. Li, W. Ding, Y. Shi, and Y. Li, "Multi-sensor based highprecision direct georeferencing of medium-altitude unmanned aerial vehicle images," International journal of remote sensing, vol. 38, no. 8-10, pp. 2577-2602, 2017.

[10] H. Li, W. Ding, X. Cao, and C. Liu, "Image registration and fusion of visible and infrared integrated camera for medium-altitude unmanned aerial vehicle remote sensing," Remote Sensing, vol. 9, no. 5, p. 441, 2017.

[11] Z. Pan, J. Lei, Y. Zhang, X. Sun, and S. Kwong, "Fast motion estimation based on content property for low-complexity h. 265/hevc encoder," IEEE Transactions on Broadcasting, vol. 62, no. 3, pp. 675-684, 2016. 